\title{
cuerpo, presencia y distancia en la enseñanza de la filosofía. exploración educativa durante el distanciamiento social.
}

\author{
nigel manchini ${ }^{1}$ \\ cfe-ces, urugray \\ orcid id: https:/ / orcid.org/0000-0002-1221-9259
}

\section{resumo}

Diversos enfoques filosóficos y científicos señalan la importancia del cuerpo para los procesos educativos y la cognición en general. La suspensión de clases y virtualización de cursos debido a la pandemia de COVID-19 ofrece una ventana para investigar esas afirmaciones en el campo concreto de la enseñanza de la filosofía. En este trabajo se presentan los resultados de una exploración educativa mixta llevada a cabo con 99 docentes de filosofía durante las primeras semanas del distanciamiento social en Uruguay. Se analizan, entre otras variables, las prácticas educativas usuales de estos/as docentes, el nivel y estrategias de trabajo durante el distanciamiento, así como sus posiciones con respecto a la educación no presencial y la importancia de la dimensión corporal. Aunque en su mayoría este grupo trabajó durante el distanciamiento social, hay escepticismo acerca de la posibilidad de enseñar filosofía de manera virtual y varios/as consideran que no podrían adaptarse. En general se considera importante la dimensión corporal en la enseñanza de la filosofía. Algunos elementos con que justifican esta importancia son la riqueza que aporta al encuentro a nivel emocional, de comunicación, de flujo de interacción y de contacto con la otredad. Estos datos permiten pensar las posibilidades y limitaciones de la virtualización de la enseñanza de la filosofía. Más en profundidad, sugieren que la enseñanza de la filosofía -incluso en las clases más tradicionales y menos corporizadas- tiene como trasfondo una compleja dinámica corporal, pocas veces explicitada. Se discute en qué sentidos puede afirmarse que la enseñanza de la filosofía es un fenómeno corporizado.

palabras clave: enseñanza de la filosofía, cuerpo, presencialidad, distanciamiento social, investigación empírica.

\section{body, presence and distance in of teaching philosophy: educational exploration during social distancing.}

\begin{abstract}
Various philosophical and scientific approaches suggest the importance of embodiment for educational processes and cognition in general. Class suspension and course virtualization during the COVID-19 pandemic offers a window to investigate these claims in the specific field of teaching philosophy. This work presents the results of an educational exploration carried out with 99 philosophy teachers during the first weeks of social distancing in Uruguay. Among other variables, the usual educational practices of these teachers, the level and strategies of work during distancing, as well as their positions with respect to distance education and the importance of body dimension are analyzed. Although most of this group worked during a period of social distancing, there is skepticism about the possibility of teaching philosophy in a virtual way;
\end{abstract}

${ }^{1}$ E-mail: nigelmanchini@gmail.com 
cuerpo, presencia y distancia en la enseñanza de la filosofía.

several teachers testified that they could not adapt. In general, the bodily dimension is considered important in the teaching of philosophy. Some elements that justify this importance are the richness that it brings to the encounter at the levels of emotion, communication, flow of interaction, and contact with otherness. These data allow us to think about the possibilities and limitations of the virtualization of the teaching of philosophy. In more depth, the data suggest that the teaching of philosophy (even in the more traditional and less embodied classes) has as background a complex body dynamics, rarely explicit. The possible senses in which the teaching of philosophy could be considered as an embodied phenomenon are discussed.

keywords: philosophy teaching; embodiment; presence; social distancing; empirical research.

\section{corpo, presença e distância no ensino da filosofia. exploração educativa durante o distanciamento social.}

\section{resumo}

Várias abordagens filosóficas e científicas apontam para a importância do corpo para processos educacionais e cognição em geral. A suspensão das aulas e a virtualização de disciplinas oferecem uma janela para investigar essas alegações no campo específico do ensino de filosofia. Este trabalho apresenta os resultados de uma exploração educacional dupla realizada com 99 professores de filosofia durante as primeiras semanas de distanciamento social no Uruguai. Entre outras variáveis, são analisadas as práticas educacionais usuais desses professores, o nível e as estratégias de trabalho durante o distanciamento, bem como suas posições em relação à educação a distância e a importância da dimensão corporal. Embora a maioria desse grupo tenha trabalhado durante o distanciamento social, há ceticismo quanto à possibilidade de ensinar filosofia de maneira virtual e vários consideram que eles não poderiam se adaptar. Em geral, a dimensão corporal é considerada importante no ensino da filosofia. Alguns elementos que justificam essa importância são a riqueza que ela traz ao encontro nos níveis emocional, de comunicação, de interação e de contato com os níveis de alteridade. Esses dados nos permitem pensar nas possibilidades e limitações da virtualização do ensino de filosofia. Em profundidade, eles sugerem que o ensino da filosofia (mesmo nas classes mais tradicionais e menos incorporadas) tem como pano de fundo uma dinâmica corporal complexa, raramente explícito.

palavras-chave: ensino de filosofia, corpo, presencialidade, distanciamento social, pesquisa empírica. 
cuerpo, presencia y distancia en la enseñanza de la filosofía. exploración educativa durante el distanciamiento social.

\section{1. introducción: la ausencia de la presencia.}

Demasiado corpóreo, limitado, compacto.

Tendré que abrir los poros y disgregarme un poco.

No digo demasiado.

Oliverio Girondo, Restringido propósito,

Persuasión de los días (1942)

Esta exploración busca obtener elementos empíricos para acercarse críticamente a la práctica de la enseñanza de la filosofía. Se asume que el análisis de un sistema en condiciones anormales puede revelar aspectos ocultos en la dinámica normal. La pandemia de COVID-19 y la consecuente suspensión de clases ofrece una oportunidad: al obligar a utilizar computadoras como medio de comunicación, enrarece el lugar del cuerpo y da una ventana para observar su rol en la enseñanza de la filosofía. El período de ausencia de la presencia puede ofrecer una instancia valiosa para "re-leernos".

Desde el enfoque corporizado de la mente se entiende que la cognición es un fenómeno complejo que involucra a la totalidad del organismo, su acción y su entorno (Varela, Thompson y Rosch, 1997; Clark y Chalmers, 1998). “La mente no está en la cabeza”, sino que emerge de la dinámica entre lo interno y lo externo (Varela, 1999). En ese sentido, el aula, como espacio físico y simbólico, estimula ciertas cogniciones y conjura otras (Foucault, 2002; Manchini, 2020a). El aula no es neutra, ni lo es la forma en que se habita ese espacio para intentar enseñar filosofía (Tourn, 2016). Tampoco lo sería el espacio -físico y simbólico- que se ocuparía en una educación virtual (Velázquez y Miraballes, 2020).

En un sentido más inmediatamente pragmático y político, esta exploración pretende aportar datos a la comunidad de docentes de filosofía. El 2020 parece un experimento mental para una clase de Didáctica de la Filosofía. Una semana después del inicio de clases se declara la Emergencia Nacional y las clases se suspenden por un 
cuerpo, presencia y distancia en la enseñanza de la filosofía.

mes. El tiempo pasa, y se hace evidente que nadie sabe con certeza cuándo ni cómo se retomarán. A veces como sugerencia y a veces como imperativo, algunas autoridades y direcciones llaman al uso de las TIC y a "virtualizar los cursos". ¿Cómo hacerlo? ¿En qué medida es posible? ¿En qué medida es deseable? ¿Es posible compensar la pérdida del encuentro, la mirada, la percepción del cuerpo? ¿Qué perdemos al perder el encuentro presencial?

Objetivos: Explorar, describir y relacionar variables pedagógico-didácticas en una muestra de 99 docentes de filosofía durante el inicio del distanciamiento social.

Sistematizar esquemáticamente las prácticas habituales de esta muestra, así como sus prácticas excepcionales durante las primeras semanas de suspensión de clases.

Indagar en el rol del cuerpo en la enseñanza de la filosofía.

Aportar elementos empíricos para el debate crítico sobre la enseñanza de la filosofía.

Antecedentes: en nuestro país se han realizado varias investigaciones cualitativas acerca de la enseñanza de la filosofía (por ej. Aguirre, 2012; Berttolini, 2012; Tourn, 2016, Gonzalez y Macedo, 2012). Se desconocen antecedentes de enfoque cuantitativo. La indagación de Manchini y Tourn (2019) es el antecedente inmediato de parte de esta investigación.

Estructura de este artículo: tras detallar el diseño metodológico en el segundo apartado (Metodología), se presentan y discuten los resultados cuantitativos (Resultados cuantitativos). En el cuarto apartado (Cuerpos en un espacio) se sintetiza parte del proceso de investigación cualitativo, intentado comprender las respuestas de los/as sujetos a través de sus propias palabras. En la Conclusión se realiza una síntesis y, a partir de esta investigación y sus limitaciones, se menciona una posible prospectiva de investigación. Se propone un debate en torno a la tesis de la corporización de la enseñanza de la filosofía y los sentidos en los cuales puede afirmársela. 


\section{2. metodologia}

Para explicitar el alcance y las limitaciones de los datos obtenidos, se realiza una breve descripción de la metodología utilizando la terminología habitual (Abero, 2015; García, 2015; Corral, 2015).

Enfoque: Se realizó un estudio exploratorio, no-experimental, transversal y mixto (cualitativo/cuantitativo).

Población y muestra: la población de este estudio $(\mathrm{N}=760)$ son los/as docentes de filosofía del Uruguay. La muestra ( $n=99)$ estuvo compuesta por 64 sujetos de género femenino, 34 de género masculino y 1 que prefirió no identificarse por género. Residen en los departamentos de Montevideo (46), Colonia (17), Canelones (15), Maldonado (4), Durazno (3), Salto (3), Florida (2), Soriano (2), Rocha (2), Paysandú (2) y San José (2). Una persona no identificó su departamento (1).

Recogida de datos: Se utilizó un muestre o no probabilístico y por conveniencia -de voluntarios-. La encuesta fue divulgada en varios medios accesibles a la población (grupos en redes sociales, correo electrónico, portales educativos) con el apoyo de la Fundación Isabel Artus, la Inspección de Filosofía del CES y la Asociación Filosófica del Uruguay. Se recogieron datos desde el 30/3 al 4/4 del 2020: por lo tanto, se refieren a las tres primeras semanas de suspensión de clase.

Instrumentos: se creó una encuesta ad hoc en la que se utilizaron tres modalidades de pregunta: Múltiple Opción (MO), Escala tipo Likert (EL) y Preguntas Abiertas (PA). Al comienzo de la encuesta se sugería un tiempo de 10-15 minutos para completarla, dando así un parámetro de extensión esperada. Previo a su difusión, el cuestionario fue evaluado por un grupo de 10 docentes de filosofía.

El cuestionario se creó y difundió en Google Forms. Los resultados se sistematizaron y analizaron usando Hojas de cálculo de Google y PSPP 1.0.1 (estadísticas descriptivas, $r$ de Pearson y $t$ de Student).

Variables: se abordaron de manera cuantitativa las siguientes variables: género, años de trabajo, autopercepción pedagógica (tradicional/innovadora), nivel de contacto durante la cuarentena, adaptabilidad a entornos virtuales, uso habitual de TIC, importancia atribuida a 
cuerpo, presencia y distancia en la enseñanza de la filosofía.

la corporalidad, viabilidad atribuida a la educación virtual en filosofía y actividades realizadas durante la cuarentena. De manera cualitativa se abordaron el rol atribuido a la corporalidad y la presencialidad, y las posibilidades y limitaciones atribuidas a la educación virtual y presencial. Se agregó una pregunta abierta donde se invitaba a compartir reflexiones acerca de la enseñanza de la filosofía durante la cuarentena.

Reporte: Este texto se centra en el reporte de los aspectos cuantitativos. Los aspectos cualitativos son utilizados primordialmente para interpretar los cuantitativos. Además se presenta una primer etapa del análisis cualitativo de las respuestas a las preguntas abiertas: a partir de la fragmentación de los datos se las codifica en 5 categorías. Para cada una de esas categorías se citan fragmentos significativos.

Limitaciones: es necesario explicitar que los resultados se refieren a esta muestra en particular y no son generalizables. Estos datos deben ser utilizados con cautela, de manera analógica, para plantear nuevas preguntas más que para dar respuestas. Las siguientes son algunas de sus limitaciones principales: el diseño es exploratorio, la muestra es no-probabilística, y el instrumento - además de ser de autoinforme- es muy general al utilizar un solo ítem por variable. A estas limitaciones debe sumarse que el análisis cualitativo se hace sin la posibilidad de "volver al campo" y sin un contexto discursivo lo suficientemente denso como para interpretar algunas expresiones. Esta exploración puede considerarse un pequeño paso en el conocimiento de este fenómeno; sin dudas, no es un punto de llegada.

\section{3. resultados cuantitativos}

A continuación se presentan los resultados para las distintas variables cuantitativas. Para simplificar la interpretación a quienes no estén familiarizados/as con el lenguaje de las metodologías utilizadas, la discusión se realizará junto con la presentación de resultados y no en otro apartado, como es habitual. 
Se divide la exposición en tres partes: en la primera (3.1) se interpretan datos acerca de los/as docentes más allá del distanciamiento social. Se analizan cuestiones como los años de trabajo,

\begin{tabular}{|c|c|c|}
\hline Variable & Media & Rango \\
\hline Años de trabajo & 12,27 (DS 8,47) & 1 año - más de 25 años \\
\hline Autopercepción & $6,41(1,59)$ & 1 (muy trad.) - 10 (muy innov.) \\
\hline Adapt. EV & $3,01(1,31)$ & $1-5$ \\
\hline Uso Hab. de TIC & $3,18(1,15)$ & $1-5$ \\
\hline Corporalidad & $4,28(1,00)$ & $1-5$ \\
\hline Viabilidad EV & $2,76(1,25)$ & $1-5$ \\
\hline \multicolumn{3}{|c|}{$\begin{array}{l}\text { Tabla 1: Resultados para las variables Años de trabajo, Autopercepción } \\
\text { Pedagógica, Adaptabilidad a Entornos Virtuales (Adapt. EV.), Uso habitual de } \\
\text { TIC, Importancia atribuida a la corporalidad (Corporalidad), Viabilidad } \\
\text { atribuida a la Educación Virtual en filosofía. }\end{array}$} \\
\hline
\end{tabular}

el género, si se consideran más o menos "tradicionales o innovadores", y qué actividades son comunes en su aula.

En la segunda parte (3.2) se analiza en qué nivel se han contactado durante este periodo y qué estrategias han utilizado o piensan utilizar para "virtualizar" sus cursos. También se analiza en qué medida los/as docentes consideran viable una educación virtual. Esos datos se discuten observando la medida en que los/as docentes utilizan las TIC, y cuánto de "adaptables" se sienten a una educación virtual. En la tercera parte (3.3) se discute la cuestión del cuerpo, haciendo dialogar estos datos con datos obtenidos en 2019.

\section{1 los/as docentes de filosofía ¿cómo se perciben y qué prácticas llevan a cabo?}

\section{años de trabajo}

En esta muestra hay sujetos que viven su primer año de docentes y otros/as que se acercan a la jubilación, siendo la media 12,27 años (Tabla 1). Es interesante señalar que los años de trabajo no tuvieron ninguna correlación estadísticamente significativa ( $p=>0.05$ ). Por lo tanto, no se cumple para esta muestra la hipótesis de que quienes tienen más años de trabajo usen menos TIC, se consideren más "tradicionales" o menos adaptables a un posible cambio en la enseñanza. 
género

Es interesante observar las distribuciones según género. Para simplificar el cuestionario, se explicitaron los géneros masculino y femenino más la opción "otro" con la posibilidad de escribir lo que se considere adecuado. Esta opción fue usada por una persona que señaló "¿Tiene sentido seguir haciendo esta pregunta?".

Reconociendo la pertinencia de la interrogante, se considera que en este estudio hay dos motivos por los cuales tuvo sentido. El primero es que esta muestra es mayormente femenina (64,6\% femenino, 34,4\% masculino), como sucede en la población nacional de docentes de filosofía (70,3\% femenino, 29,7\% masculino), y como es habitual en los/as profesionales de la educación. El segundo es que existe una diferencia pequeña pero estadísticamente significativa por género al respecto de la percepción de las propias clases como tradicionales o innovadoras. Quienes se declaran femeninas se consideran más innovadoras (media 6,69) que quienes se declaran masculinos (media 5,87).

Estos datos aportan información sobre tendencias en la división del trabajo y los roles sociales. La distinción aún parece aportar, tanto para interpretar la realidad como para modificarla. Así como hay diferencias basadas en el género al respecto del trabajo, la economía y el poder en general, parece que también las hay sobre el enseñar filosofía.

\section{autopercepción pedagógica}

Se pidió a los/as sujetos que indiquen cómo consideran su práctica docente en una línea del 1 al 10, siendo 1 "muy tradicional" y 10 "muy innovadora". Como se observa en la Tabla 1, esta muestra tiende a considerarse más innovadora que tradicional, siendo la puntuación media 6,41. De hecho, ningún sujeto se identificó con puntuación 1, y solamente 5 sujetos se identificaron con puntuación 2 o 3. Es llamativo observar que también en el otro polo encontramos una medida similar: solamente 6 sujetos se identifican con puntuación 9 y 10. La moda es 7. Pareciera que entre estos/as docentes de Filosofía se valora la innovación, pero también la moderación. 
Es interesante observar que hay una correlación débil pero estadísticamente significativa $(r=0,31, p=0,002)$ entre la autopercepción pedagógica y el uso habitual de las TIC. Sea por el contexto actual o por una asociación endémica, los/as docentes tomaron el uso de la tecnología como una de las medidas de su tradicionalismo/innovación.

\section{prácticas habituales}

Para evaluar en qué medida es posible enseñar filosofía sin la instancia de encuentro presencial, es vital saber qué actividades son habituales en un aula de filosofía. Se abordó esa pregunta analizando la pregunta: "¿qué tipo de actividades son comunes en su aula?". Se contaron y agruparon las menciones de los docentes, tal como se representa en la Tabla 2 y la Figura 1. En este grupo de docentes la mención más frecuente es

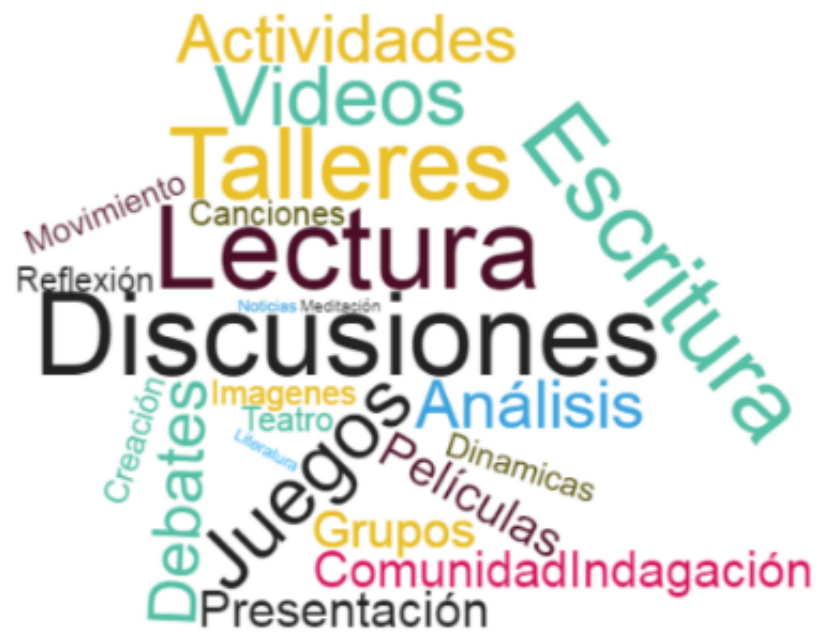

Figura 1. Panorama de actividades usuales en las aulas de los/as docentes encuestados. El tamaño de la palabra se corresponde con el número de menciones. a la lectura, las discusiones, la escritura y los talleres.

Es importante la prudencia a la hora de interpretar estos datos. Primero, porque los "tipos de actividades" mencionados son muy variopintos: desde conceptos muy generales como "Talleres" o "Actividades" hasta referencias metodológicamente más definidas como "Comunidad de indagación" o recursos como "Canciones" o "Imágenes".

Además, la mención frecuente no implica la realización frecuente. Por ejemplo, la alta mención a "Talleres" y la poca mención de "Presentación" no implica necesariamente que los talleres sean más usuales que las clases expositivas. 
cuerpo, presencia y distancia en la enseñanza de la filosofía.

por otro lado, pueden aplicar sesgos de deseabilidad.

Muchas de estas actividades no son actividades que necesariamente impliquen un uso del espacio presencial y del cuerpo: la lectura y la visualización de videos puede realizarse en una pantalla, las presentaciones pueden grabarse, las discusiones pueden tenerse a través de foros, etc. Sin embargo, en las preguntas abiertas abundan las referencias a una pérdida de calidad en las discusiones y en las exposiciones, debido al empobrecimiento de la comunicación noverbal y paraverbal $y$, consecuentemente, de aspectos emocionales vinculados a estas actividades.

Esto es consistente con los datos de la

\begin{tabular}{|l|l|}
\hline Tipo de actividad & Menciones \\
\hline Lectura & 71 \\
Discusiones & 63 \\
Escritura & 30 \\
Talleres & 27 \\
Juegos & 23 \\
Videos & 18 \\
Debates & 17 \\
Actividades & 16 \\
Análisis & 15 \\
Películas & 8 \\
Presentación & 8 \\
Comunidad de indagación & 7 \\
Grupos & 5 \\
Canciones & 4 \\
Imágenes & 4 \\
Creación & 3 \\
Dinámicas & 3 \\
Reflexión & 3 \\
Movimiento & 2 \\
Teatro & 2 \\
\hline Tabla 2. Conteo y agrupación de palabras \\
claves usadas para describir actividades \\
habituales en el aula de filosofía \\
\hline
\end{tabular}
“Indagación sobre la corporeidad en el aula" (Manchini y Tourn, 2019). En ella, se encuestó a 44 docentes de filosofía acerca del rol de la corporeidad en el aula. Frente a la pregunta de cómo incluían la dimensión corporal en el aula, el 75\% afirmó prestar atención a lo que el cuerpo comunica, y el 63,5\% declaró realizar modificaciones en el espacio (como tener clases afuera, sentarse en ronda, etc.); solamente un 27\% afirmó realizar actividades que tuvieran al cuerpo como elemento central (danza, teatro, meditación, realización audiovisual, etc.).

Considerando esto, parece natural echar de menos el encuentro presencial aun cuando no se realicen actividades centradas en lo corporal. El cuerpo es protagonista en talleres y actividades, pero además la dinámica de miradas, gestos, posturas y entonaciones es el escenario sin el cual la palabra y el concepto no pueden ser protagonistas. 


\section{2 el distanciamiento social y la enseñanza de la filosofia}

\section{contacto y trabajo durante el distanciamiento social}

Como se observa en la

Figura 2, más de la mitad de

los/as docentes declaran haber virtualizado parte de su curso, mientras que otro tercio afirma haber enviado una actividad. Solamente 3 docentes afirman no tener contacto con sus estudiantes, mientras que 3 afirman tener contacto pero no haber enviado ninguna

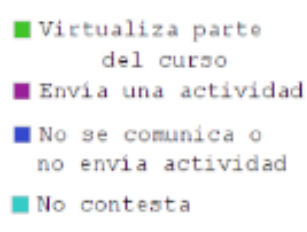
actividad.

En términos cuantitativos se puede decir que la afirmación "las clases se suspendieron" es imprecisa o falsa, al menos para esta muestra.

\section{recursos de educación a distancia utilizados}

Pero ¿qué significa en términos cualitativos "virtualizar" un curso de filosofía?

¿Qué tipo de actividad envían los/as docentes de filosofía?

Resulta conveniente leer las respuestas a la pregunta ¿Qué recursos de educación a distancia ha utilizado o piensa utilizar?

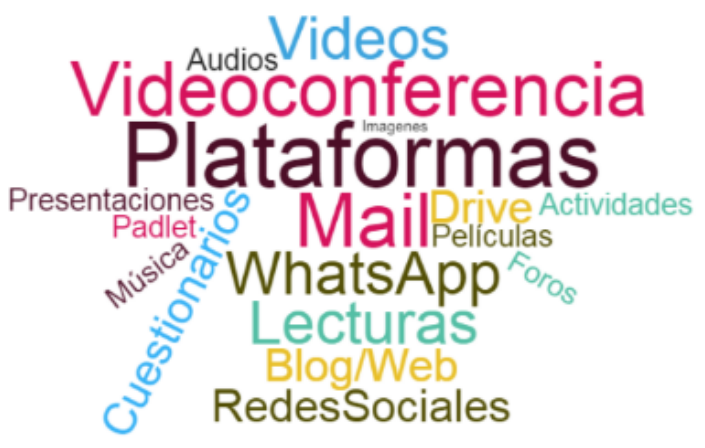

Figura 3. Medios de comunicación, recursos y actividades utilizadas durante primeras tres semanas. El tamaño de la palabra se corresponde con el número de menciones 
cuerpo, presencia y distancia en la enseñanza de la filosofía.

Recuérdese que la ausencia de mención no significa necesariamente que esa actividad no se realice. Por ejemplo, solamente hay 15 menciones a la lectura, pero probablemente sea casi omnipresente en las actividades enviadas.

Las respuestas se sintetizan en la Figura 3 y la tabla 2.

En lo referente a los medios de comunicación, el uso de las diversas plataformas educativas es el medio más popular, junto con medios de comunicación que frecuentemente acompañan los cursos presenciales: mail, WhatsApp, blogs, páginas web y Google Drive. Entre las plataformas, la más mencionada es CREA, junto con Moodle, Edmodo y Ceibal.

Algunos/as docentes explicitaron qué tipo de actividad realizaron. La más normal es el uso de videos, ya sea grabados por los/as docentes o seleccionados. Es frecuente, también, la mención a las lecturas, cuestionarios (de preguntas abiertas o cerradas), películas

\begin{tabular}{|l|l|l|l|}
\hline Medios de comunicación & & Tipos de actividades & \\
\hline Plataformas (CREA, Ceibal, & & Videos & 24 \\
Moodle, etc.) & 59 & Lecturas & 15 \\
Mail & 31 & Cuestionarios & 10 \\
Videoconferencia & 29 & Películas & 6 \\
WhatsApp & 28 & Presentaciones & 5 \\
Blog/Web & 14 & Actividades varias & 5 \\
Drive & 8 & Foros & 4 \\
Otras redes sociales (FB, IG, & 6 & Padlet & 3 \\
Twitter) & & Música & 2 \\
& & Audios & 1 \\
& & Imágenes & 1 \\
\hline
\end{tabular}

Tabla 3. Sistematización de la respuestas a la pregunta "¿Qué recursos de educación a distancia ha utilizado o piensa utilizar?". Los números significan menciones; cada sujeto pudo mencionar más de una actividad.

y presentaciones. En algunos casos, la discusión de esos materiales se realiza en foros $\mathrm{u}$ otros espacios virtuales que permiten la interacción.

\section{el uso de las tic y la adaptación a un entorno virtual}

Se pidió a los/as docentes que cuantifiquen del 1 al 5 su nivel de acuerdo con la afirmación "En el caso de que fuera necesario, me adaptaría bien a una modalidad nopresencial." Como se ve en la Tabla 1, la media es de 3,01. Más de un tercio (37 docentes) puntúan 1 y 2, expresando que les sería difícil adaptarse, mientras que 26 docentes expresan neutralidad frente a su posible adaptación. Es importante tener este dato en cuenta en un contexto donde la enseñanza virtual puede verse más o menos impuesta. 
Es revelador observar que existe una correlación estadísticamente significativa entre la adaptabilidad percibida y el uso anterior de las TIC $(r=0,43, p=0.000)$. Es posible aventurar que el poco uso de las TIC sea un factor que lleva a los/as docentes a dudar de su adaptación a la educación no-presencial. Sin embargo, que la correlación sea moderada (y no alta o perfecta) indica que hay otras variables en juego.

\section{enseñanza virtual de la filosofia}

"En un escenario donde todos los problemas técnicos estuvieran solucionados, sería posible enseñar filosofía de manera virtual". Según se ve en la Tabla 1, la media de acuerdo a esta afirmación fue de 2,76.

46 docentes se ubicaron en polo escéptico (puntuando 1 y 2). Es interesante notar que ese escepticismo no debe atribuirse a debilidades técnicas ("no todos/as tienen computadoras”, “la plataforma colapsa”, "se corta la videoconferencia”, etc.). En el otro polo, 26 docentes afirmaron su creencia en una posible educación virtual puntuando 4 y 5 .

Cabe notar que hay una correlación moderada-alta y estadísticamente significativa $(r=0.59, \mathrm{p}=0.000)$ entre esta respuesta y la respuesta por la adaptabilidad a un entorno no virtual. Podría interpretarse que los/as docentes definen cuánto de viable es la educación virtual proyectando cuánto “se ven" a sí mismos/as trabajando en un entorno virtual (y/o viceversa).

\section{3 la corporeidad transparente}

¿cuánto es considerada la dimensión corporal por el común de los/as docentes?

Es interesante analizar las respuestas en torno a la importancia atribuida al cuerpo poniéndolas en diálogo con otras respuestas sobre el mismo tópico en un período anterior. 
En 2019 se realizaron las jornadas académicas "Cuerpo, filosofía y educación", y en ese contexto se realizó la "Indagación sobre la corporeidad en el aula" (Manchini y Tourn, 2019). Esa indagación empírica con docentes de filosofía $(\mathrm{n}=44)$ tuvo características similares a esta, pero con una muestra más sesgada -integrada en su mayoría por docentes que asistieron a las jornadas, y por lo tanto, tenían interés en prácticas educativa que incluyen la dimensión corporal-.

En esa indagación, se le preguntó a los/as docentes “ ¿Cuánto de relevante te parece la dimensión corporal en el aula de filosofía?", en una escala de 1 (nada relevante) a 10 (muy relevante). Como era esperable, esa muestra consideraba la dimensión corporal como muy relevante: la media fue de 8,27 (DS=1,96). Sin embargo, cuando se le consultó "Según tu percepción, ¿cuánto es considerada la dimensión corporal por el común de lxs docentes de filosofía?" la media fue de 3,48 (DS=1,92). En otras palabras, los/as docentes de esa indagación consideraban que lo corporal es relevante en su aula, pero no en el aula de la mayoría de sus colegas. Lúdicamente, se le llamó a esto efecto Kafka: los/as docentes de esa muestra se sentían "bichos raros".

\section{¿un error de estimación?}

En la presente exploración se preguntó el grado de acuerdo con la afirmación "la dimensión corporal es importante en mi forma de enseñar filosofía", en una escala de 1 (nada de acuerdo) a 5 (muy de acuerdo). La media fue 4,28 (DS=1), expresando que la dimensión corporal es, en promedio, considerada como muy importante.

Entonces, podría decirse que los/as sujetos de la indagación de 2019 se equivocaron. Estimaron que "el común de los/as docentes" consideraba poco la dimensión corporal (3,48 en un rango del 1-10). Sin embargo, cuando se le preguntó a un grupo de docentes que no parece tener un sesgo hacia lo corporal, la mayoría dice que la dimensión corporal es muy importante en su forma de enseñar filosofía. Por otro lado, alguien podría afirmar que fueron los/as sujetos de 2020 quienes se equivocaron: por verse privados del cuerpo y la presencialidad, exageran la importancia que le dan a la dimensión corporal. 


\section{polisemia de "dimensión corporal"}

Podría argumentarse, sin embargo, que ambos conjuntos de sujetos están en lo correcto, y que la aparente contradicción descansa en la polisemia de la expresión “dimensión corporal".

A fines de 2019, cuando se realizó la "Indagación sobre la corporeidad en el aula", se presuponía el trabajo presencial. Cuando afirmaron que "el común de los/as docentes" considera poco la dimensión corporal, el encuentro en un espacio físico se daba por obvio. Se pensaba más bien en cómo se habitaban el cuerpo y el espacio: ¿Se cuestionaban los usos hegemónicos del cuerpo? ¿Se criticaban las asignaciones basadas en el género? ¿Se pensaban propuestas didácticas que incluyeran la implicación sensorio-motriz?

En otras palabras: cuando los/as sujetos de 2019 estimaron que sus colegas consideran poco la dimensión corporal, no se discutía que las didácticas "descorporizadas" también necesitaban la presencia corporal en un espacio físico. Los/as docentes de 2020, por el contrario, definen cuánto de importante es la dimensión corporal teniendo por parámetro la suspensión de clases, el distanciamiento social y la posible virtualización de los cursos. El cambio de contexto moduló el significado de "considerar la dimensión corporal". Como señala un sujeto de este estudio en su reflexión libre: "nunca me había percatado tanto de la incidencia del encuentro cara a cara" (s5).

Podría argumentarse que el distanciamiento social pone sobre la mesa una corporeidad transparente (concreta, pero difícil de ver).

Lo que se hace evidente no parece ser una corporización didáctica explícita: no se parece a la educación corporizada (Nguyen y Larson, 2015; Shapiro y Stolz, 2019) de quien planifica propuestas que involucren la interacción, el movimiento, la manipulación, etc. Más bien, lo ausente es algo que permanecía implícito. 


\section{4. cuerpos en un espacio}

Hay distintas formas de explicitar la corporeidad implícita en el aula. ¿Cuál eligieron los/as docentes de este estudio? Excede los límites de este texto realizar un análisis cualitativo exhaustivo. Aun así, con ánimo de invitar a la reflexión, se presentan algunas de las respuestas a las preguntas: "Para la enseñanza de la filosofía ¿Considera importante que los cuerpos (docentes/estudiantes) compartan un espacio físico? ¿Por qué?".

Para presentar estas ideas se citará texto de las respuestas, organizado en torno a ciertos tópicos que fueron frecuentemente referidos. Como bien saben quiénes enseñan filosofía, la selección de texto implica una lectura subjetiva, y siempre deben dejarse afuera elementos significativos. Los datos cuantitativos anteriormente compartidos pretenden tener una "impersonalidad" -que no es objetividad- de la cual la siguiente selección carece completamente.

\section{la riqueza del encuentro}

En el aula de filosofía hay más que comunicación verbal. Como señala la s60 “No es posible hacer filosofía sin ese encuentro, donde todo habla, las miradas, los silencios, las palabras, la invención del momento, lo impredecible. Nada de esto es posible sin vernos, olernos, escucharnos, sentirnos".

Una valoración frecuente fue que una parte importante de la riqueza del encuentro se perdía con la mediación de computadoras. Como señala el s9: "La mejor enseñanza es aquella que la mediatización no puede lograr al velar lo expresivo, lo actitudinal, al cercenar buena parte de lo que somos" (s9). La s84, en la misma línea, señala: "las mediaciones tecnológicas reducen el campo de nuestra relación a lo consciente presente, al resultado visible de nuestros procesos de elaboración; simplifican los devenires de nuestro pensamiento y esconden por disimulo, la conmoción que un pensar experiencial nos genera."

\section{el cuerpo comunica y crea un ambiente afectivo}

"Somos más que cerebros en una cubeta" dice la s69. 
"La presencia corporal trae un montón de ingredientes didáctico/pedagógicos" (s12), entre otras cosas, porque "puede ser muy importante para transmitir aspectos emotivos y vivenciales de la actitud filosófica" (s17). La "lectura de gestos" (s22) permite "percibir el grado de interés, de adhesión o rechazo" (s17) y a partir de ahí "estar presente y acompañar los procesos de aprendizaje" (s38).

Pero, más allá de esta interpretación de los gestos, "hay algo insustituible en el encuentro, una energía corporal, una espontaneidad" (s36) en el "cuerpo a cuerpo" y "el voz a voz" (s13) que facilita la "vivencia de hacer filosofia" (s19). En otras palabras, la presencia corporal no solo da mensajes sino que también crea un clima afectivo: "la motivación afectiva del encuentro y de estar con otres es insustituible" (s21). Muchos/as encuentran que "crear un ambiente que haga entrar en situación a los alumnos (...) se dificulta mucho más a distancia" (s89).

\section{amor por la sabiduría}

El encuentro de los cuerpos en un espacio es propio de la filosofía. Como señala el s10 "la filosofía nace en el ágora, en el diálogo, por lo tanto sin estos elementos sería muy difícil hacer una filosofía vivencial (quizá la más efectiva) para intentar generar la actitud filosófica. La presencia física del otro, con su gestualidad y postura ayuda a entender que la alteridad es esencial en esta disciplina: porque despierta emociones de respeto, solidaridad, empatía, que son imprescindibles para la enseñanza de las humanidades. La filosofía es un acto de amor que sólo puede darse de forma humana".

Esa emocionalidad de la disciplina se vincula también con su enseñanza. La enseñanza de la filosofía "es vivencial, hay un componente emocional y hasta diría artístico en el trabajo en el aula" (s5). ¿Pero no lo hay en toda enseñanza? Como señala la s20 " $\mathrm{La}$ didáctica es cuerpo y es mirada, es lectura de gestos, es amor vivenciado en el vínculo físico".

\section{el tiempo y la interacción}

Como señala la s66: "Se siente extraño trabajar en plataforma y parece estar "tirando una botella al mar", estar esperando una respuesta". 
cuerpo, presencia y distancia en la enseñanza de la filosofía.

La clase es un momento específico, limitado en el tiempo. "Cuando estamos en el aula (no solo el salón), estamos ahí, es el momento de la clase", dice la s29. El encuentro simultáneo de los cuerpos es importante porque dinamiza el "flujo de la interacción" (s87). El encuentro "en tiempo real hace que el ejercicio sea dinámico y profundo"; es "la inmediatez del diálogo y el intercambio de pareceres" (s98) la que permite "el encuentro y desencuentro de ideas, de pensamientos, el ir y venir de los diferentes logos, inquietudes, problemas y posibles soluciones" (s96).

\section{la apertura a la otredad}

El encuentro "permite que surjan otros emergentes que se presentan cuando se genera un vínculo de confianza (...) muy difícil de generar online" (s3). El aula habilita y estimula la "integración y disrrupción de la otredad" (s20). Como dice la s32: "En el aula presencial no se puede evitar lo distinto, estamos obligados a compartir con quienes nos interesa y con quienes no".

La s12 señala un aspecto importante que emerge del encuentro con los otros/as: la improvisación: "valoro el encuentro, las miradas, el estar en un mismo espacio físico interactuando en el momento e improvisando el curso de la clase por lo que acontece más allá de lo planeado". Como señala la s13, la presencialidad "permite que la relación estudiante/docente sea más espontánea y permeable a las diversas situaciones".

\section{5. conclusión: síntesis y discusión}

\section{1 sintesis}

En este texto se describieron, analizaron y relacionaron una serie de variables. Habiéndose descrito las limitaciones metodológicas, se enfatiza en que no es posible generalizar estos datos: cuando se dice "los/as docentes" se habla de este grupo de docentes. Teniendo eso en mente, sintetizar algunas características de esta muestra puede sernos útil para pensar por analogía, reflexionar y formular nuevas preguntas.

a. ¿Qué hacen estos/as docentes de filosofía habitualmente? Los/as docentes de filosofía consideran sus prácticas más "innovadoras" que "tradicionales". ¿Qué 
podemos encontrar cuando les preguntamos por esas prácticas? Según se infiere, la lectoescritura es casi omnipresente, así como la discusión -ya sea en formato de debate, de comunidad de indagación u otros-. Hay también un conjunto importante de docentes que realizan juegos y talleres. Hay un lugar para la exposición y análisis de conceptos, pero también lo hay para reflexionar desde las artes (cine, imagen, música, literatura, teatro), tanto consumiendo como creando.

b. ¿Cómo trabajó este grupo de docentes durante las primeras tres semanas del distanciamiento social? La inmensa mayoría de este grupo realizó algún tipo de “intervención educativa virtual". Este estudio no es confiable para generalizar, pero nos da motivos para proponer la hipótesis de que sucedió una virtualización de facto. Sin embargo, debemos ponderar el contenido de esa afirmación: en muchos casos, esa “virtualización” no pasa de enviar un texto o el link de un vídeo y un cuestionario. Hay sin embargo, una cantidad significativa de docentes que han realizado videoconferencias, han filmado sus clases o han utilizado otros recursos que hablan de una "virtualización” más significativa. Las plataformas educativas han sido la forma más popular de contactar con los/as estudiantes, pero un caudal importante de información circula a través de WhatsApp, mails, blogs y páginas web.

c. ¿Es viable una enseñanza de la filosofía no presencial? Según la opinión mayoritaria de esta muestra, debemos ser escépticos/as sobre esa posibilidad. Aún en un contexto donde se solucionaran los problemas técnicos, una parte significativa de la enseñanza de la filosofía se perdería. Esta creencia se encuentra relacionada con la percepción que muchos/as tienen de que no se adaptarían con facilidad a un entorno no presencial de aprendizaje.

d. ¿Qué importancia tiene lo corporal en la enseñanza de la filosofía? Las respuestas de este grupo aportan evidencia a la hipótesis de que lo corporal tiene un rol central en la enseñanza de la filosofía. Además, permiten proponer la hipótesis de que ese rol va más allá de las prácticas educativas corporizadas (que incluyen movimiento, manipulación y acción corporal): incluso un aula donde reina la quietud y el silencio está imbricada con un acontecer a nivel corporal, es un acontecer a nivel 
cuerpo, presencia y distancia en la enseñanza de la filosofía.

corporal. Aun cuando no es abordado explícitamente, el cuerpo es un protagonista implícito.

Las respuestas de los/as docentes dan algunas guías de qué mirar para entender el rol de la presencia y la corporeidad en el aula. La riqueza del encuentro (con sus sonidos, miradas, olores) es difícilmente alcanzable en una comunicación mediada por computadoras. Una parte importante de lo que se pierde está vinculado con el contenido emocional, tanto en un sentido interpretativo ("lectura de gestos") como en un sentido productivo ("crear un ambiente"). Esto se vincula con el aspecto emocional de la filosofía: es un tipo de relación (de amistad, de amor, de deseo) con el conocimiento y la sabiduría. El encuentro, además, permite un "flujo de la interacción" fluido y controlado. Sin embargo, hay un límite a ese control ya que en el encuentro la otredad irrumpe, rompiendo la linealidad y permitiendo la aparición de emergentes.

\section{2 más allá de esta investigación: la enseñanza de la filosofía como fenómeno corporizado}

Por las limitaciones antes señaladas, estos datos no son una base sólida para teorizar. Sin embargo, se pueden usar sus debilidades como plataforma para la reflexión. Parte de las limitaciones van más allá de esta investigación concreta, y serán comunes a cualquier investigación similar; en este contexto, se invita a la comunidad de investigadores/as, filósofos/as y docentes a trabajar sobre esas limitaciones.

\section{prospectiva empírica}

En lo referente a la metodología, algunas de las limitaciones podrían superarse -con tiempo y recursos- en investigaciones posteriores: se podría buscar una población representativa, se podría triangular el autoinforme con análisis documental $\mathrm{u}$ observación directa, y se podrían establecer mecanismos de comunicación con los/as sujetos para enriquecer y validar el análisis cualitativo de sus respuestas. Existe, sin embargo, una limitación importante que persistiría: la inexistencia de instrumentos validados para investigar la enseñanza de la filosofía y el rol del cuerpo en ella. Si la 
comunidad de investigadores/as reconoce la importancia de la investigación empírica de la enseñanza de la filosofía, la construcción y estandarización de instrumentos es un paso importante a dar.

\section{debate conceptual}

Sin embargo, hay limitaciones importantes que pertenecen al ámbito conceptual. En lo que resta se analizará una. Como se señaló en la parte 3.3, una de las grandes dificultades para abordar la corporeidad en la educación es la polisemia de expresiones como "dimensión corporal", "cuerpo", "corporalidad", "corporeidad", etc.. Más en concreto, la afirmación "la enseñanza de la filosofía es un fenómeno corporizado" puede ser verdadera (o falsa) en múltiples sentidos. Desentrañar esos múltiples sentidos parece una tarea digna para la filosofía de la enseñanza de la filosofía.

Al igual que en la discusión sobre la corporización de la cognición (Wilson y Foglia, 2017), podemos postular una tesis de la corporización de la enseñanza de la filosofía: Aspectos significativos de la enseñanza de la filosofía son corporizados, en tanto son profundamente dependientes del cuerpo físico de los/as agentes y sus dinámicas.

Formular esta tesis puede ayudar a centrar la discusión, pero no elimina la polisemia; parafraseando a Aristóteles, "la corporización se dice de muchas maneras". A modo de reflexión final, se proponen cinco sentidos en los cuales puede afirmarse que la enseñanza de la filosofía es corporizada. Para nada se busca realizar una lista exhaustiva: se pretende, apenas, aportar ejemplos de la polisemia mencionada y comenzar una discusión. Será una tarea colectiva ponderar el valor de estos ejemplos y definir de manera precisa cuáles son los sentidos en que la corporización puede afirmarse.

Un sentido cognitivo. Desde la perspectiva de la cognición corporizada, se puede afirmar que la enseñanza de la filosofía es corporizada en el mismo sentido en que lo es cualquier otro fenómeno que involucre procesos cognitivos. Desde este enfoque, la cognición “depende de las experiencias originadas en la posesión de un cuerpo con diversas aptitudes sensorio-motrices, (...) encastradas en un contexto biológico, psicológico y 
cuerpo, presencia y distancia en la enseñanza de la filosofía.

cultural más amplio" (Varela et al., 1997, p.203). Este sentido es abordado con respecto a la educación en Manchini (2020a; 2020b) y en Shapiro y Stolz (2019). En Varela et al. (1997), Varela $(2005,1996)$, Wilson (2002), Wilson y Foglia (2017) pueden consultarse visiones más generales.

Un sentido comunicacional. La enseñanza de la filosofía es un fenómeno social y comunicacional. En tanto la cognición social y la comunicación son dependientes del cuerpo, también la enseñanza de la filosofía lo es. Desde variopintas tradiciones, los trabajos de Ekman (2003), Benenzon (2000), Watzlawick (2009) y Rizzolatti y Craighero (2004) dan cuenta de que aspectos importantes de la comprensión de otras personas y la comunicación suceden a nivel paraverbal y no-verbal. Este sentido parece ser el referido por los/as docentes al hablar de la importancia del cuerpo para comunicar y crear un ambiente afectivo. También, parte de la "riqueza del encuentro" parece referir a este nivel analógico de la comunicación.

Un sentido fenomenológico. Es usual distinguir entre el cuerpo como proceso fisiológico (o cuerpo objeto, Körper) y el cuerpo vivido (Leib) (Merleau-Ponty, 1994; Thompson, 2004). El cuerpo vivido "no es el cuerpo objeto visto con un microscopio o rayos$x$, es el cuerpo que ve o que existe en el acto de ver" (Gallagher, (1986, 140, tr.pr.). Todas nuestras vivencias son "en primera persona" y desde nuestra corporeidad; en palabras de Foucault (2008, p.11): "mi cuerpo es lo contrario de una utopía: es aquello que nunca acontece bajo otro cielo". En este sentido, la enseñanza de la filosofía es un fenómeno corporizado en tanto los/as estudiantes y docentes experimentan la clase desde su corporeidad. El trabajo de Tourn (2016) podría inscribirse en esta perspectiva. Considerando esto, cuando los/as docentes sienten que la mediación tecnológica puede "cercenar buena parte de lo que somos", esto debe entenderse no solo en relación a lo que no pueden hacer, sino también a lo que no pueden experimentar.

Un sentido político-institucional. La institución educativa, en tanto institución disciplinaria, implica "un arte del cuerpo humano", siendo "un mecanismo de poder que lo explora, lo desarticula y lo recompone" (Foucault, 2002, p.141). Aunque la escuela moderna parezca ser una escuela sin cuerpo, esta "descorporización" es solamente aparente 
(Manchini, 2020a; Scharagrodsky, 2007). Como señaló Althusser, la ideología reproducida en la escuela no tiene existencia ideal, sino material: "es material, en tanto esas ideas son actos materiales insertos en prácticas materiales, reguladas por rituales materiales" (Althusser, 2003, p.50). La enseñanza de la filosofía es corporizada en tanto ocurre en instituciones corporizadas. Cuando los/as sujetos de esta investigación manifiestan extrañar el aula, podría sospecharse que lo que realmente extrañan es esta matriz moderna -esta lectura puede ser un poco reduccionista, pero es sugerida por Cal (2020) en su comentario a esta investigación.

Un sentido pedagógico. Desde distintos marcos teóricos se propone la necesidad de una pedagogía corporizada, que ubique la manipulación y la acción como centrales incluso para la enseñanza de conocimientos con un alto componente abstracto como la matemática o la lectura (Manchini, 2020b; Nguyen y Larson, 2015; Shapiro y Stolz, 2019). Vale la pena ver que mientras los cuatro primeros sentidos corresponderían a lo que se llamó "corporización implícita", esta última línea de pensamiento apuntaría a una "corporización explícita" (ver 3.3).

Solo la investigación conceptual y empírica podrá completar y precisar esta esquemática e incompleta lista. Pero, sobre todo, el debate debe articularse con la práctica: no se trata solo de interpretar la corporeidad, sino de transformarla. Las instituciones educativas son las herederas de una tradición de corporización, ¿No es posible cambiar esa matriz? ¿No son posibles otras formas de ser cuerpos y habitar los espacios educativos?

Al principio de este texto, se dijo que el funcionamiento anormal puede hacer evidente aquello que el funcionamiento normal oculta. Pedagogo cruel, el presente parece mostrarnos la importancia del cuerpo distanciándonos de él. Pero, ¿Cuánta distancia es posible? Quizás la situación excepcional lleve a que se exploren las prácticas educativas más etéreas e incorpóreas. Pero nunca será demasiado: siempre, y en muchos sentidos, somos cuerpos. 
cuerpo, presencia y distancia en la enseñanza de la filosofía.

\section{referencias}

Abero, L. (2015). Técnicas de recogida de datos. En L. Abero, L. Berardi, A. Capocasale, S. García y R. Rojas (2015). Investigación educativa: abriendo puertas al conocimiento. Montevideo: Contexto.

Aguirre, M. (2012). El diálogo con los textos filosóficos en el aula de enseñanza media. Estudio sobre las buenas prácticas en la enseñanza de la filosofía. Cuadernos de Investigación Educativa, 3(18). 65-97.

Althusser, L. (2003). Ideología y aparatos ideológicos del Estado. Freud y Lacan. Buenos Aires: Nueva Visión.

Benenzon, R. (2000). Modelo musicoterapéutico de Benenzon. En M. Betés (comp.), Fundamentos de musicoterapia, (329-349). Madrid: Morata.

Berttolini, M. (2012). Investigación sobre la enseñanza de la filosofía. Linhas 13(1). 88-107

Cal, D. (2020). Reflexiones sobre la enseñanza de la FIlosofía en confinamiento social. Portal Uruguay Educa. https://uruguayeduca.anep.edu.uy/recursos-educativos/4757

Clark, A. y Chalmers, D. (1998). The extended mind. Analysis 58 (1).

Corral, Y. (2015). Procedimientos de muestreo. Revista Ciencias de la educación 26 (46). 151167.

De Sousa Santos, B. (2020). La cruel pedagogía del virus. Buenos Aires: CLACSO.

Ekman, P. (2003). Emotions revealed. Nueva York: Times Edition.

Foucault, M. (2002). Vigilar y castigar. Buenos Aires: Siglo XXI

Foucault, M. (2008). Topologías. Fractal, 48 (12), 39-50. Recuperado de http:/ / hipermedula.org/wp-

content/uploads/2013/09/michel_foucault_heterotopias_y_cuerpo_utopico.pdf

Gallagher, S.(1986) Lived body and environment. Research in Phenomenology, 16. 139-170

García, S (2015). Aspectos metodológicos de la investigación cualitativa. En L. Abero, L. Berardi, A. Capocasale, S. García y R. Rojas (2015). Investigación educativa: abriendo puertas al conocimiento. Montevideo: Contexto.

González, I. y Macedo, C. (2012). ¿Cómo aprenden a evaluar los profesores?. CFE, IPA. http://repositorio.cfe.edu.uy/handle/123456789/616

Manchini, N. (2020a). La mente en el cuerpo: diálogo entre la cognición corporizada y la educación. Dialektika, 2(4), 1-16.

Manchini, N. (2020b). Mente, cuerpo y acción: caminatas entre las ciencias cognitivas corporizadas y la educación. Artículo entregado para publicación.

Manchini, N. y Tourn, J. (2019). Indagación sobre la corporeidad en el aula. En Fundación Isabel Artus y Asociación Filosófica del Uruguay (Organización). “Cuerpo, Filosofía y Educación", Jornadas académicas llevadas a cabo en CeRP SW, Colonia del Sacramento, noviembre de 2019.

Merleau-Ponty, M. (1994). Fenomenología de la percepción. Buenos Aires: Planeta.

Nguyen, D. J., y Larson, J. B. (2015). Don't Forget About the Body: Exploring the Curricular Possibilities of Embodied Pedagogy. Innovative Higher Education, 40(4), 331-344.

Rizzolatti, G., y Craighero, L. (2004). The Mirror-Neuron System, Annual Review of Neuroscience, 27: 169-192.

Scharagrodsky, P. (2007). El cuerpo en la escuela. Buenos Aires: MECT: Explora. Pedagogía. Recuperado de http:/ / www.memoria.fahce.unlp.edu.ar/libros/pm.869/pm.869.pdf 
Shapiro, L., y Stolz, S. A. (2019). Embodied cognition and its significance for education. Theory $\mathcal{E}$ Research in Education, 17(1), 19.

Thompson, E. (2004). Life and mind: from autopoiesis to neurophenomenology. A tribute to Francisco Varela. Phenomenology and the Cognitive Sciences, 3. 381-398.

Tourn, J. (2016). Cuerpo y enseñanza de la filosofía. Trabajo Final de Maestría. Instituto CLAEH, Montevideo.

Varela F. (1999) Steps to a science of interbeing: Unfolding the dharma implicit in modern cognitive science. En: Watson G., Bachelor S. y Claxton G. (eds.) The psychology of awakening. Rider, New York: 71-89.

Varela, F. (1996). Ética y acción. Santiago de Chile: Dolmen.

Varela, F. (2005). Conocer. Las ciencias cognitivas: tendencias y perspectivas. Barcelona: Gedisa.

Varela, F., Thompson, E. y Rosch, E. (1997). De cuerpo presente: las ciencias cognitivas y la experiencia humana. Barcelona: Gedisa.

Velazquez, S. y Miraballes, G. (8/2/2020). Cómo educar después de la pandemia. La diaria. https://ladiaria.com.uy/articulo/2020/4/como-educar-despues-de-la-pandemia/

Watzlawick, P. (2009) Teoría de la comunicación humana. Barcelona: Herder.

Wilson, M. (2002). Six views on embodied cognition. Psychonomic Bulletin E Reviews 9(4). 625636.

Wilson, R. y Foglia, L. (2017). Embodied Cognition. En The Stanford Encyclopedia of Philosophy, Edward N. Zalta (ed.).

Agradecimientos: se agradece especialmente a Lucía Cristina, Nicolás Olivera, Carolina Clavero y Agustina Pons, compañeros/as de la Fundación Isabel Artus.

También a Christian Burgues y Adelina Pintos de AFU y Mario López y Lilyán García de Inspección de Filosofía por el apoyo institucional. Gracias a Janett Tourn y Marolyn Regueiro por el apoyo y la disposición a trabajar juntos/as. También a Walter Kohan y los/as evaluadores/as de Childhood \& Philosophy por sus útiles comentarios.

recibido en: 24.04 .2020

aprobado en: 24.06 .2020 